\title{
Fiebre de origen desconocido. Forma de presentación de los linfomas malignos
}

\author{
V. ROCA CAMPAÑÁ, H. RODRÍGUEZ SILVA
}

Servicio de Medicina Interna. Hospital Hermanos Ameijeiras. Habana, Cuba

\author{
MALIGNANT LYMPHOMAS PRESENTING AS FEVER OF UNKNOWN \\ ORIGIN
}

\section{RESUMEN}

Objetivo: Los linfomas malignos constituyen la primera causa de fiebre de origen desconocido (FOD) dentro de la categoría de las neoplasias. Se realizó este estudio con el objetivo de determinar los hallazgos clínicos y aquellas investigaciones que contribuyeron al diagnóstico de dichos tumores.

Método: Se realizó un estudio descriptivo-prospectivo de 18 pacientes hospitalizados para el estudio de una FOD clásica en los que se estableció el diagnóstico de linfoma maligno durante los años 2000 al 2005.

Resultados: Más de la mitad de los casos (55\%) se encontraba en estadio IV. Se detectó perdida de peso en 13 pacientes $(72 \%)$ y adenopatías periféricas en la mitad de los casos. El ultrasonido abdominal evidenció alteraciones sugestivas de linfoma maligno en el 55\% de los casos. La biopsia de adenopatías periféricas o profundas estableció el diagnóstico en 8 pacientes (44\%) y la de médula ósea en 6 pacientes (33\%). Hubo 4 pacientes fallecidos (22\%) en los que el diagnóstico solo se pudo establecer en la necropsia.

Conclusiones: La mayoría de los linfomas malignos que debutaron por FOD se encontraba en estadio avanzado. La perdida de peso y las adenopatías periféricas fueron las manifestaciones clínicas más frecuentes. El ultrasonido abdominal, la biopsia de adenopatías y la de médula ósea fueron los procederes de mayor utilidad diagnóstica.

PALABRAS CLAVE: Fiebre de origen desconocido. Linfoma de Hodgkin. Linfoma no Hodgkin.

\section{ABSTRACT}

Objective: The main cause of fever of unknown origin (FUO) in the diagnostic category of neoplasms are malignant lymphomas. This study was done to describe the clinical features and the investigation results which diagnostic contribution.

Method: Between 2000 and 2005 a prospective and descriptive study was conducted in 18 patients hospitalized for studying a classical FUO who were diagnosed as having a malignant lymphoma.

Results: More than half of all patients (55\%) were in stage IV. The weight loss and the peripheral lymphadenopathy were found in $13(72 \%)$ and 9 patients (50\%), respectively. Abdominal ultrasonography had a diagnostic contribution in 55\% of the patients. The malignant lymphoma diagnostic was made by peripheral or deep lymphadenopathy biopsy in 8 patients (44\%). The bone marrow biopsy established diagnostic in 6 patients (33\%). In four patients (22\%) the diagnosis was made at the autopsy.

Conclusions: Most of patients with malignant lymphomas presenting as FUO had an advanced disease. The weight loss and the peripheral lymphadenopathy were the most frequent clinical features. Abdominal ultrasonography, lymphadenopathy biopsy and bone marrow biopsy were the procedures with more diagnostic utility.

KEY WORDS: Fever of unknown origin. Hodgkin's lymphoma. NonHodgkin's lymphoma.

Roca Compañá V, Rodríguez. Silva H. Fiebre de origen desconocido. Forma de presentación de los linfomas malignos. An Med Interna (Madrid) 2007; 24: 531-534.

\section{INTRODUCCIÓN}

La predilección de los linfomas malignos por asociarse a un síndrome febril prolongado y el retraso que puede existir en su diagnostico han sido reconocidos desde hace varias décadas (1).

En 1961 Petersdorf y Beeson publican los criterios para definir una FOD:

a) Fiebre mayor de 38,3 grados centígrados en varias ocasiones; b) Fiebre prolongada por más de 3 semanas; y c) Diagnóstico no precisado después de haber realizado investigaciones durante 1 semana de hospitalización (2). La etiología de la FOD comprende cinco grandes categorías. Infecciones, neoplasias, enfermedades inflamatorias no infecciosas, misceláneas y un grupo que permanece sin diagnóstico (3).

Las neoplasias constituyen la tercera o la segunda causa de FOD y dentro de esta categoría los linfomas malignos representan alrededor del $50 \%$ de los casos, de acuerdo a cinco grandes series publicadas de diversas áreas geográficas (4-8). De igual forma en un estudio que realizamos en nuestro país durante el período de 1988 a 1992, donde evaluamos 105 pacientes con FOD, encontramos un predominio manifiesto de los linfomas malignos (76\%) en la categoría de las neoplasias (9). En este trabajo reportamos un estudio prospectivo-descriptivo de 18 casos de linfomas malignos Hodgkin y no Hodgkin que ingresaron por FOD durante los años 2000 al 2005. 


\section{MATERIAL Y MÉTODO}

Se realizó un estudio prospectivo-descriptivo en el Hospital "Hermanos Ameijeiras" durante los años 2000 al 2005, ambos inclusive.

Se seleccionaron 18 pacientes que habían sido hospitalizados en el Servicio de Medicina Interna para el estudio de una FOD, en los que se estableció el diagnostico de linfoma maligno como causa del síndrome febril. Para ser incluidos en el estudio los pacientes tenían que cumplir con los criterios de FOD establecidos por Petersdorf y Beeson (2). Se excluyeron aquellos casos que tenían diagnostico previo de linfoma maligno y que ingresaron por una FOD.

Se confeccionó una base de datos donde se recogió de forma estandarizada la siguiente información: a) tipo de linfoma maligno y estadio; b) manifestaciones clínicas: síntomas, presencia de adenopatías, esplenomegalia, hepatomegalia; c) resultado de las siguientes investigaciones: hemograma con diferencial, eritrosedimentación, conteo de plaquetas, TGP, TGO, FAL, LDH, Rx de tórax (AP), ultrasonido de abdomen, TAC de tórax, TAC de abdomen, biopsias de ganglio linfático, médula ósea, hígado, bazo u otra localización; y d) fallecidos y causas de muerte.

\section{RESULTADOS}

La edad promedio de los 18 casos estudiados fue de $48 \pm$ 17,4 años (osciló entre 24 y 80 años). Ocho pacientes $(44,4 \%)$ eran del sexo femenino y diez $(55,5 \%)$ del sexo masculino. Del total de casos estudiados 8 tenían enfermedad de Hodgkin (44\%) y 8 linfoma no Hodgkin (44\%), en dos casos se confirmó el diagnostico de linfoma maligno pero no se pudo clasificar su variedad. De este total de 18 pacientes, 3 se encontraban en estadio I (16\%), 2 en estadio II (11\%), 2 en estadio III (11\%) y 11 en estadio IV (55\%).

Los síntomas más frecuentes fueron: pérdida de peso en 13 pacientes $(72 \%)$, astenia en 11 pacientes $(61 \%)$ y anorexia en 9 pacientes $(50 \%)$. El tipo de fiebre de Pel-Ebstein se observó en 4 casos (22\%), solo 3 pacientes (16\%) tuvieron sudoración nocturna (Tabla I).

El examen físico demostró la presencia de adenopatías periféricas en 9 casos $(50 \%)$, con un tamaño inferior a $1 \mathrm{~cm}$ en 3 casos y entre $1-3 \mathrm{~cm}$ en 6 casos. En 3 pacientes las adenopatías periféricas no estuvieron presentes al momento del ingreso, sino que aparecieron evolutivamente, uno de ellos cuando ya habían transcurrido 6 meses del debut del síndrome febril. En 5 casos existía hepatoesplenomegalia (27\%), en 3 hepatomegalia aislada (16\%) y en 2 esplenomegalia aislada (11\%) (Tabla I).

Los exámenes de laboratorio evidenciaron la presencia de anemia en 13 pacientes (72\%) y leucopenia en 3 (16\%). Uno de estos pacientes, previo a la leucopenia, presentó una leucocitosis de $50 \times 10^{9} / 1$ con un $88 \%$ de eosinófilos, que fue interpretado inicialmente como una leucemia mieloide crónica variante eosinofílica. Posteriormente por biopsia de médula ósea se pudo establecer el diagnostico de linfoma no Hodgkin.

La trombocitopenia estuvo presente en 2 pacientes (13\%). Todos los pacientes tuvieron eritrosedimentación acelerada y de ellos en 11 casos (61\%) estuvo por encima de $100 \mathrm{~mm} / \mathrm{h}$. Con relación a las pruebas funcionales hepáticas hubo elevación de la TGP y la TGO en 6 pacientes (37\%). La fosfatasa alcalina estuvo elevada en 8 pacientes $(66 \%)$, de un total de 12 en que se realizó este estudio. La LDH estuvo elevada en 5 pacientes $(55 \%)$ de un total de 9 casos estudiados.
TABLA I

MANIFESTACIONES CLÍNICAS EN PACIENTES CON FOD POR LINFOMA MALIGNO $(n=18)$

\begin{tabular}{lcc}
\hline Síntomas & $N^{0}$ de casos & $\%$ \\
\hline Pérdida de peso & 13 & 72 \\
Astenia & 11 & 61 \\
Anorexia & 9 & 50 \\
Tos seca & 5 & 27 \\
Sudoración nocturna & 3 & 16 \\
Disnea & 2 & 11 \\
Prurito generalizado & 1 & 5 \\
Cefalea & 1 & 5 \\
Dolor torácico & 1 & 5 \\
Vómitos & 1 & 5 \\
Desorientación & 1 & 5 \\
Artralgias & 1 & 5 \\
Diarreas & 1 & 5 \\
Signos & & \\
Adenopatías & 9 & 50 \\
Hepatoesplenomegalia & 5 & 27 \\
Hepatomegalia & 3 & 16 \\
Esplenomegalia & 2 & 11 \\
\hline
\end{tabular}

TABLA II

RESULTADO DE INVESTIGACIONES DE LABORATORIO EN PACIENTES CON FOD POR LINFOMA MALIGNO

\begin{tabular}{|c|c|c|c|}
\hline $\begin{array}{l}\text { Parámetro de } \\
\text { laboratorio }\end{array}$ & $\begin{array}{l}N^{0} \text { de casos } \\
\text { detectados }\end{array}$ & $\begin{array}{l}\text { Diagnóstico } \\
N^{0} \text { casos }\end{array}$ & $\%$ \\
\hline $\begin{array}{l}\text { Anemia }(\mathrm{Hb}<11 \mathrm{~g} / \mathrm{l}) \\
\text { Leucopenia }\end{array}$ & 13 & 18 & 72 \\
\hline $\begin{array}{l}\text { (leucocitos < 4,0 x 109\%/) } \\
\text { Trombocitopenia }\end{array}$ & 3 & 18 & 16 \\
\hline $\begin{array}{l}\left.\text { (plaquetas }<150 \times 10^{9} / \mathrm{l}\right) \\
\text { Eritrosedimentación acelerada }\end{array}$ & 2 & 15 & 13 \\
\hline (VSG > $20 \mathrm{~mm} / \mathrm{h}$ ) & 18 & 18 & 100 \\
\hline ASAT > $40 \mathrm{Ud} / /$ & 6 & 16 & 37 \\
\hline $\begin{array}{l}\text { ALAT > } 40 \mathrm{Ud} / \mathrm{l} \\
\text { Fosfatasa alcalina }\end{array}$ & 6 & 16 & 37 \\
\hline (FAL > $279 \mathrm{Ud} / \mathrm{l})$ & 8 & 12 & 66 \\
\hline $\mathrm{LDH}>460 \mathrm{U} / \mathrm{l}$ & 5 & 9 & 55 \\
\hline
\end{tabular}

A todos los pacientes se les realizó Rx de tórax. Se encontró ensanchamiento mediastinal en 4 casos (22\%) y derrame pleural y pericárdico en 1 caso $(5 \%)$. La tomografía axial computarizada (TAC) de tórax se le realizó a 6 pacientes y se confirmó la presencia de adenopatías mediastinales en 3 de los 4 casos que tenían ensanchamiento del mediastino por $\mathrm{Rx}$ de tórax. A todos los pacientes se les realizó ultrasonido (US) de abdomen y en 10 (55\%) se encontraron alteraciones que permitían la sospecha de linfoma maligno, estas fueron: hepatoesplenomegalia 3 casos, esplenomegalia 2 casos, adenopatías profundas 2 casos, esplenomegalia con adenopatías profundas 1 caso, hepatomegalia con esplenomegalia nodular 1 caso y nódulos hepáticos 1 caso. En 4 de estos pacientes estas alteraciones aparecieron en el curso evolutivo de la enfermedad. La TAC de abdomen se realizó en 4 casos (22\%), y en 2 de ellos se puso en evidencia nueva información que no había sido detectada en el US previo, sobre todo en un caso que demostró la presencia de nódulos hipodensos en bazo e hígado. 
El diagnóstico de linfoma en vida se estableció por biopsia en 14 casos $(77 \%)$, esto se distribuyó de la siguiente forma: biopsia de ganglios en 8 pacientes $(44 \%)$ y biopsia de sitios extranodales (médula ósea, hígado) en 7 pacientes (38\%). En un paciente el diagnóstico se confirmó tanto por la biopsia de ganglios como por la biopsia de médula ósea. La biopsia de ganglios se realizó en 5 casos en ganglios periféricos y en 3 casos fue mediante procederes más invasivos: dos por mediastinoscopía y uno por excéresis de ganglios intraabdominales mediante laparotomía. A través de la biopsia de sitios extranodales se detectaron 6 pacientes con infiltración linfomatosa de médula ósea, 3 por enfermedad de Hodgkin y 3 por linfoma no Hodgkin, en un paciente se determinó infiltración hepática a partir de una biopsia en cuña durante una laparotomía.

Se realizó biopsia de médula ósea en 15 pacientes y biopsia hepática en 11, incluyendo los casos fallecidos, estas tuvieron un rendimiento diagnóstico de un $40 \%$ y un $9 \%$ respectivamente (Tabla III).

Durante el estudio fallecieron 4 pacientes (22\%), en los que el diagnóstico se determinó en la necropsia, 3 de ellos en relación directa con la enfermedad por infiltración cardiaca, pulmonar y cerebral. El cuarto fallecimiento fue a consecuencia de una hemorragia gastrointestinal por ulcera péptica. En estos 4 fallecidos la biopsia de médula ósea (4 casos) y la biopsia hepática (1 caso) no mostraron infiltración tumoral.

TABLA III

RENDIMIENTO DIAGNÓSTICO DE BIOPSIA DE SITIOS EXTRANODALES EN PACIENTES CON FOD POR LINFOMA MALIGNO

\begin{tabular}{lccc}
\hline $\begin{array}{l}\text { Estudio } \\
\text { histológico }\end{array}$ & $\begin{array}{c}N^{0} \text { casos } \\
\text { estudiados }\end{array}$ & $\begin{array}{c}\text { Diagnóstico } \\
N^{0} \text { casos }\end{array}$ & $\%$ \\
\hline Biopsia médula ósea & 15 & 6 & 40 \\
Biopsia hepática & 11 & 1 & 9 \\
\hline
\end{tabular}

\section{DISCUSIÓN}

Se estudiaron 18 pacientes hospitalizados por FOD en los que se estableció el diagnóstico de linfoma maligno como causa del síndrome febril. Se encontró igual proporción de pacientes con enfermedad de Hodgkin (44\%) y con linfoma no Hodgkin (44\%). Más de la mitad de los casos se encontraban en estadio IV. La mayoría de las FOD ocasionadas por neoplasias se deben a los linfomas malignos, sobre todo cuando se encuentran en estadio avanzado $(1,5,10-12)$.

Los síntomas presentes fueron muy variados, con un franco predominio de los síntomas generales: pérdida de peso $(72 \%)$, astenia $(61 \%)$ y anorexia $(50 \%)$.

El tipo de fiebre de Pel-Ebstein solo estuvo presente en 4 casos, todos con un síndrome febril con una evolución prolongada que osciló entre 4 meses y 2 años, tres de estos casos tenían una enfermedad de Hodgkin (13).

Las adenopatías periféricas se detectaron en el 50\% de los casos, con un tamaño no mayor de $3 \mathrm{~cm}$ de diámetro, en un tercio de los casos estas aparecieron evolutivamente durante el estudio de la FOD. Esto confirma, como se ha afirmado, la importancia del examen físico repetido en los pacientes con FOD (14). La esplenomegalia y/o la hepatomegalia estuvieron presentes en más de la mitad de los casos (55\%).
En un estudio realizado por Iikuni Yayoi y colaboradores en 153 pacientes con FOD las adenopatías constituyeron el signo más frecuente en los tres principales grupos de FOD (infecciones, enfermedades del tejido conectivo/vasculitis, neoplasias), y se reportó hepatoesplenomegalia en el $42 \%$ de 12 pacientes con FOD por linfoma maligno (6).

La eritrosedimentación acelerada $(100 \%)$ y la anemia $(72 \%)$ fueron los hallazgos más frecuentes en los estudios de laboratorio clínico. Esto se corresponde con lo reportado por Esposito y Gleckman, donde todos los pacientes con FOD por linfoma maligno tuvieron anemia y eritrosedimentación acelerada $(12,15)$. De igual forma en otro estudio de 48 pacientes con FOD por neoplasias se evidenció anemia en el $89,6 \%$ y eritrosedimentación acelerada en el 77,1\% (16). El número de pacientes con LDH alta y con LDH normal fue muy similar. Debemos considerar que esta investigación solo se realizó en la mitad de los casos.

La radiografía de tórax (AP) mostró ensanchamiento mediastinal en el $22 \%$ de los casos. Un estudio multicéntrico de 167 pacientes con FOD reportó la contribución del Rx de tórax en el diagnostico de diversas entidades, entre ellas los linfomas malignos (17).

La TAC de tórax confirmó en 3 de 4 pacientes que el ensanchamiento mediastinal se debía a la presencia de adenopatías. La TAC de tórax es un estudio indispensable para evaluar el mediastino, particularmente cuando el diagnostico de linfoma maligno es probable $(18,19)$.

El ultrasonido abdominal constituyó un medio diagnostico útil, en el $55 \%$ de los casos puso en evidencia algunas alteraciones que permitieron sospechar el diagnostico de linfoma. En ocasiones estas alteraciones se detectaron en ultrasonidos evolutivos, lo que pudiera estar en relación con la experiencia del investigador, o con la evolución de la enfermedad.

En un estudio multicéntrico de 167 pacientes con FOD se reconoce la contribución del ultrasonido abdominal para determinar la etiología de la FOD (17). Si tenemos en cuenta este reporte y el nuestro, así como su bajo costo al compararlo con otros estudios imagenológicos, no hay dudas que el ultrasonido abdominal constituye, como se ha afirmado, una investigación de primera elección en el estudio de los pacientes con FOD $(18,19)$.

La TAC de abdomen solo se realizó en 4 pacientes, en 2 de ellos aportó información complementaria a la reportada en el ultrasonido abdominal.

La TAC de abdomen es una de las investigaciones que debe realizarse durante el estudio de la FOD y es particularmente útil ante la sospecha de un proceso linfoproliferativo por ser una investigación que pone en evidencia signos orientadores para realizar biopsia. Se reporta un rendimiento diagnostico de un $19 \%$ en la FOD $(20,21)$. Por todo esto el ultrasonido abdominal y la TAC de abdomen deben considerarse como investigaciones que aportan información complementaria de gran valor en el estudio de la FOD $(17,18,21)$.

La biopsia de adenopatías periféricas y profundas permitió arribar al diagnostico de linfoma maligno en el $44 \%$ de los casos. No hay dudas acerca de la importancia de la biopsia de ganglios linfáticos cuando la FOD se acompaña de adenopatías, incluso si hay que recurrir a técnicas invasivas. Se señala una utilidad diagnostica de un 50\% cuando se realiza excéresis de adenopatías para su estudio en pacientes con FOD $(6,17)$.

La biopsia de sitios extranodales (médula ósea, hígado) confirmó el diagnóstico en el $38 \%$ de los pacientes. Este resultado es expresión de una enfermedad en estadio avanzado, lo que suele ocurrir en pacientes con linfoma maligno que debutan con 
una FOD (10). La médula ósea fue el principal sitio extranodal con utilidad diagnostica (40\%), en 2 pacientes fue necesario repetir dicha biopsia para poder arribar al diagnostico, como ha sido reportado en otra publicación (11). Este proceder cuando se realiza en pacientes con FOD tiene un rendimiento diagnostico de alrededor de un $14 \%(11,17,22)$. En el caso de nuestro estudio el porcentaje de positividad de la biopsia de médula ósea fue muy superior (40\%), como era de esperar por tratarse de una población seleccionada de pacientes con FOD por linfoma.

La presencia de pancitopenia, anemia y/o trombocitopenia pueden ser indicadores de infiltración de médula ósea por linfomas malignos en el curso de una FOD, aunque su ausencia no excluye que se realice biopsia de médula ósea $(1,10,11,17)$. En los pacientes que el diagnostico se realizó a través de biopsia de médula ósea, la anemia fue la alteración hematológica en sangre periférica más frecuente.

La biopsia hepática tuvo un rendimiento diagnostico bajo, solo fue positiva en un caso. En 10 de los 11 pacientes que se realizó este proceder había alguna alteración de las pruebas funcionales hepáticas y/o evidencias de hepatomegalia o nódulos hepáticos por el examen físico o el ultrasonido abdominal. Otros reportes también señalan una utilidad diagnostica baja de este proceder cuando se estudian pacientes con FOD de diversas etiologías o en pacientes con FOD por linfoma maligno $(1,17)$. Este examen se considera una investigación de segunda o tercera línea durante el estudio de la FOD. La mayoría de los autores señala un rendimiento diagnostico de aproximadamente un $14 \%(18,20,22)$.
La laparotomía contribuyó al diagnostico en 2 pacientes, uno de ellos tenía esplenomegalia que se extendía hasta flanco izquierdo y no se había establecido la causa de la fiebre, se realizó esplenectomía y se hallaron adenopatías intraabdominales (no detectadas previamente), el estudio histológico de los ganglios permitió establecer el diagnóstico de linfoma maligno no Hodgkin. El otro caso fue una paciente que presentó una colecistitis aguda durante el estudio de la FOD, en el acto quirúrgico se tomó biopsia hepática en cuña lo que permitió determinar el diagnóstico de linfoma maligno no Hodgkin. En esta paciente previo a la cirugía se había detectado una FAL más de dos veces por encima del nivel normal y tenía una biopsia hepática por laparoscopia negativa.

Con el avance experimentado en los estudios de imágenes y en técnicas de toma de biopsias se ha reducido la necesidad de realizar laparotomía. Sin embargo en ocasiones este proceder es necesario y útil, particularmente cuando se basa en resultados positivos de estudios de imágenes previos y resulta imposible la biopsia por ultrasonido o cirugía de mínimo acceso $(12,18,22-25)$.

En relación con los 4 pacientes fallecidos debemos señalar que: la causa de la muerte se debió a la extensión del linfoma maligno en 3 pacientes, el tiempo de hospitalización fue inferior a 30 días en 3 casos, en todos los pacientes se realizó algún proceder de toma de biopsia en vida (médula ósea, hígado) a pesar de esto el diagnostico solo pudo establecerse en la necropsia.

\section{Bibliografía}

1. Larson EB, Featherstone HJ, Petersdorf RG. Fever of undetermined origin: Diagnosis and follow up of 105 cases, 1970-1980. Medicine 1982; 61: 269-92

2. Petersdorf RG, Beeson PB. Fever of unexplained origin: Report on 100 cases. Medicine 1961; 40: 1- 30.

3. Knockaert DC, Vanderschueren S, Blockmans D. Fever of unknown origin in adults: 40 years on. J Intern Med 2003; 253: 1-15.

4. Ramos Rincon JM, Ramos Guevara R, Ferrero Huerta F. Fiebre de origen desconocido en Medicina Interna. Experiencia de autores españoles durante 20 años. An Med Interna (Madrid) 1997; 14: 585-92.

5. de Kleijn EMHA, Vandenbroucke JP, van der Meer JWM and The Netherlands FUO Study Group. Fever of unknown origin (FUO).I. A prospective multicenter study of 167 patientes with FUO, using fixed epidemiologic entry criteria. Medicine 1997; 76: 392-400.

6. Iikuni Y, Okada J, Kondo H, Kashiwazaki S. Current fever of unknown origin 1982-1992. Intern Med 1994; 33: 67-73.

7. Tabak F, Mert A, Celik AD, Ozaras R, Altiparmak MR, Ozturk R, et al. Fever of unknown origin in Turkey. Infection 2003; 6: 417-20.

8. Molina-Gamboa J, Rivera-Morales I, Camacho-Mezquita E, Ponce-deLeon S. The changing spectrum of fever of unknown origin: trends and comparison with previous series at the Salvador Zubiran National Institute of Nutrition. Rev Invest Clin 1994; 46: 177-85.

9. Cruz Peña LA, Rodríguez Silva H, Pérez Caballero D. Fiebre de origen desconocido: Revisión de 105 pacientes. Rev Cubana Med 1995; 34: 1-10.

10. Arnow PM, Flaherty JP. Fever of unknown origin. Lancet 1997; 350: 575-80.

11. Hirschmann JV. Fever of unknown origin in adults. Clin Infect Dis 1997; 24: 291-302.

12. Woolery A, Franco FR. Fever of unknown origin. Keys to determining the etiology in older patients. Geriatrics 2004; 59: 41-5.

13. Beutler B, Beutler SM. Patogenia de la fiebre. En: Cecil. Tratado de Medicina Interna. 20 ed. McGraw-Hill Interamericana, 1996.p. 1770-3.
14. Mackowiak AP, Durack DT. Fever of unknown origin. Mandell: Principles and Practice of Infectious Diseases, 5ta ed. 2000 Churchill Livingstone pag 623-33.

15. Esposito AL, Gleckman RA. Fever of unknown origin in the elderly. J Am Geriatr Soc 1978; 26: 498-505.

16. Wei W, Hesheng L. Malignancy in chinese adults presenting as fever of unknown origin. Int J Clin Pract 2003, 57: 508-12.

17. de Kleijin EMHA, van Lier HJJ, van der Meer JWM, and the Netherlands FUO Study Group. Fever of unknown origin (FUO). II. Diagnostic procedures in a prospective multicenter study of 167 patients. Medicine 1997; 76: 401-14

18. Knockaert DC. Diagnostic strategy for fever of unknown origin in the ultrasonography and computed tomography era. Acta Clin Belg 1992; 47: $100-16$.

19. Knockaert DC, Vanneste LJ, Bobbaers HJ. Fever of unknown origin in the elderly patients. J Am Geriatr Soc 1993; 41: 1187-92.

20. Mourad O, Palda V, Detsky AS. A comprehensive evidence-based approach to fever of unknown origin. Arch Intern Med 2003; 163: 545-51.

21. Quinn MJ, Sheedy PF, Stephens DH, Hattery RR. Computed tomography of the abdomen in evaluation of patients with fever of unknown origin. Radiology 1980; 136: 407-11.

22. Nolan SM, Fitzgerald FT. Fever of unknown origin. The general internist's approach. Postgraduate Medicine 1987; 81: 190-205.

23. Ozaras R, Celik AD, Zengin K, Mert A, Ozturkk R, Cicek Y, Tabak E. Is laparotomy necessary in the diagnosis of fever of unknown origin? Acta Chir Bel 2005; 105: 89-92.

24. Arch-Ferrer JE, Velázquez-Fernández D, Sierra-Madero J, Lopez-Karpovitch X, Ángeles-Ángeles A, Gamino R, et al. Laparoscopic approach to fever of unknown origin. Surg Endosc 2003; 17: 494-397.

25. Takahashi T, Herrera MF, Onuma L, Calva JJ, Sánchez-Mejorada G, Hurtado R, et al. Diagnostic laparotomy in fever of unknown origin. Rev Invest Clin 1991; 43: 25-30. 